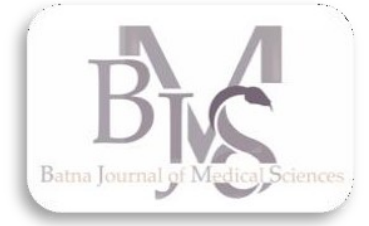

Cabinet libéral de Pédiatrie, 35 Rue De Mostaganem, Oran Algérie

\section{Correspondance à :}

Abdelmadjid BENMANSOUR

benmansour_b@yahoo.com

DOI :https://doi.org/10.48087/ B]MStf.2015.2110

Il s'agit d'un article en libre accès distribué selon les termes de la licence Creative Commons Attribution International License (CC BY 4.0), qui autorise une utilisation, une distribution et une reproduction sans restriction sur tout support ou format, à condition que l'auteur original et la revue soient dûment crédités.

\title{
Les mucopolysaccharidoses
}

\author{
Mucopolysaccharidoses
}

\section{Abdelmadjid Benmansour}

\section{RÉSUMÉ}

Les Mucopolysaccharidoses (MPS) sont des maladies héréditaires dues à un défaut enzymatique \{l'origine de dépôts de glycosaminoglycanes (mucopolysaccharides) au niveau des lysosomes. Ces dépôts vont se retrouver au niveau de tous les organes de façon plus ou moins importante avec des manifestations plus ou moins précoces et plus ou moins parlantes selon le taux de l'activité résiduelle au niveau des organes atteints.Il y a 7 différents types de mucopolysaccharidoses; Certains signes cliniques sont retrouvés en commun chez la plupart de ces maladies en particulier les organomégalies et l'atteinte osseuse. D'autres sont plus spécifiques chez certaines avec une atteinte cérébrale prédominante telle la MPS III (maladie de San Filippo). Elles font l'objet de très nombreuses recherches et des traitements sont possibles avec des résultats encourageants surtout si le traitement est entrepris précocement. Il y a des traitements adjuvants : physiothérapie, médicamenteux, chirurgie cardiaque, oculaire selon le cas. Il existe un traitement spécifique : la greffe de moelle pour certaines des MPS, et un traitement de substitution enzymatique également pour certaines MPS.Plus récemment, des molécules chaperonnes ont été utilisées pour obtenir une augmentation de l'activité résiduelle et dernièrement un traitement par thérapie génique est en cours dévaluation dans plusieurs centres.

Mots clés : mucopolysaccharidoses ; maladies lysosomales ; déficit enzymatique ; atteinte multiviscérale ; atteinte cérébrale.

\begin{abstract}
Mucopolysaccharidoses (MPS) are hereditary diseases due to an enzyme deficiency, leading to deposits of glycosaminoglycanes (mucopolysaccharides), in lysosomes. These deposits will be found in all organs in a more or less important way, with more or less early and more or less severe clinical manifestations according to the level of the residual activity in the involved organs. There are 7 different types of mucopolysaccharidoses; some types are found in common in the majority of the diseases, especially the bone involvement and organomegaly. Others are more specific in some types, with a predominant cerebral involvement like in the MPS III (San Filippo disease). These conditions are subject to many research studies and treatments are possible with encouraging results, mostly if the treatment is early initiated. Other adjunctive treatments may be used, such as physiotherapy, symptomatic drugs and heart and eyes surgery. A specific treatment is available: the bone marrow transplant for some types of MPS, and the enzyme substitution in others. Recently, chaperone molecules have been used in order to obtain an increase in the residual activity, and more recently, gene therapy is being evaluated in many centres.
\end{abstract}

Keywords: mucopolysacharidoses; lysosomal diseases; enzyme deficiency; multiorgan deficiency; cerebral involvement.

\section{Pour citer l'article :}

Benmansour A. Mucopolysaccharidoses. Batna J Med Sci 2015;2(1):40-44. https://doi.org/10.48087/ B]MStf.2015.2110

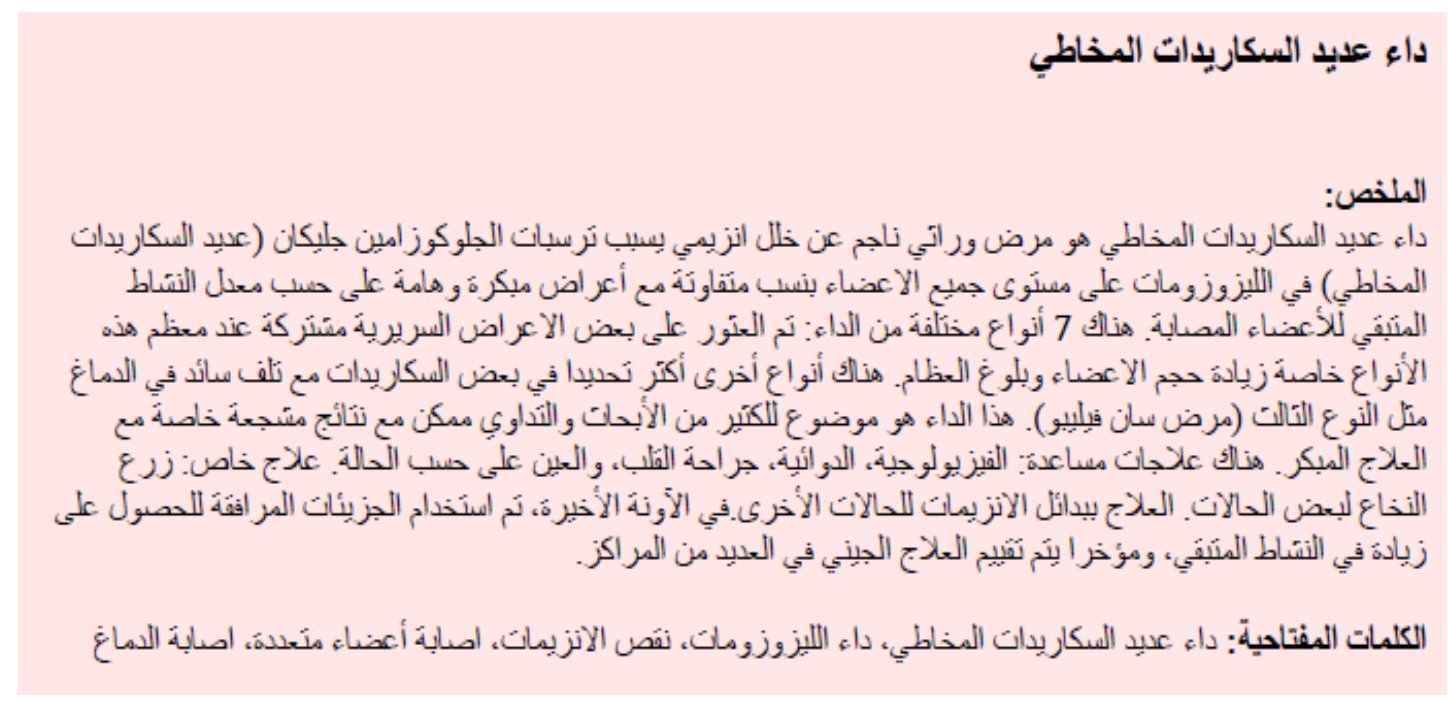




\section{DÉFINITION}

Ce sont des maladies héréditaires rares à transmission autosomique récessive \{l'exception de la maladie de Hunter qui se transmet selon le mode lié $\left\{\mathrm{l}^{\prime} \mathrm{X}\right.$ [1]. La plupart sont multisystémiques, même si l'atteinte d'un système peut être prédominante.Elles sont dues \{ un déficit d'une enzyme lysosomale impliquée dans la dégradation des glucosaminoglycanes (Héparane sulfate, Kératane sulfate, Dermatane sulfate et Chondroïtine sulfate) [1]. Ces glucosaminoglycanes proviennent elles-mêmes de la dégradation de protéoglycanes qui sont des constituants importants de la matrice extracellulaire (sauf l'acide hyaluronique) et du tissu conjonctif de tout l'organisme (figure 1).
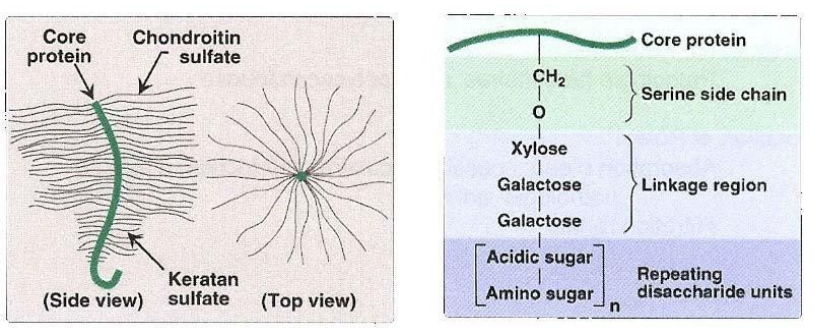

Figure 1 : Schémas extraits du cours de biochimie des glucides pour CPEM Pr. André Le Treut de la Faculté de Médecine de Rennes avec son aimable permission[2].

N'étant pas dégradés, ces éléments vont s'accumuler au niveau des lysosomes et être ainsi \{ l'origine d'un dysfonctionnement cellulaire, tissulaire et enfin de tous les organes. Les processus physiopathologiques sont encore incomplètement compris et font l'objet de recherche poussée[3].Dans toutes les Mucopolysaccharidoses on retrouve des formes atténuées et des formes sévères. Il ya un continuum entre toutes ces formes (Tableau 1).

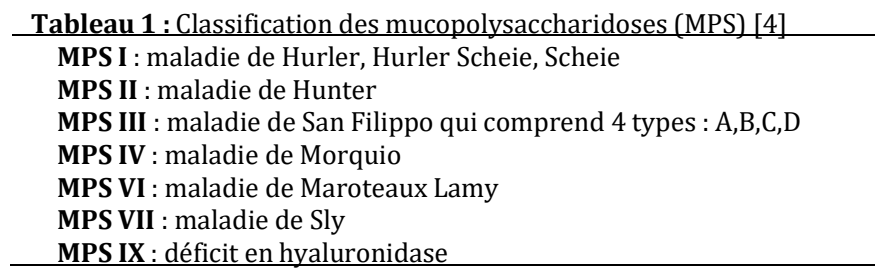

\section{MANIFESTATIONS CLINIQUES COMMUNES AUX MPS}

- Hernie ombilicale ou inguinale souvent récurrente ;

- Cou court ;

- Anomalies du squelette, gibbosité, pieds varus ou valgus ;

- infections ORL répétées ;

- faciès à traits grossiers :

- limitation articulaire modérée ;

- foie et rate augmentés de volume ;

- valvulopathie mitrale ou aortique avec ou sans cardiomyopathie.

\section{Manifestations tardives :}

- Faciès aux traits grossiers avec macroglossie, gencives épaissies Dents espacées ;

- Main en griffe ;
- Cheveux épais en chaume ;

- Hydrocéphalie ;

- hépato-splénomégalie ;

- surdité ;

- opacification cornéenne, glaucome, dégénérescence rétinienne ;

- apnées du sommeil ;

- Manifestations squelettiques dysostoses multiples ;

- Arthropathies : limitation articulaire sévère.

Les signes radiologiques ne sont pas pathognomoniques mais peuvent orienter de façon significative le diagnostic des MPS:

- selle turcique en oméga ;

- élargissement costal, des métas et des phalanges ;

- vertèbre cunéiforme, et vertèbres ovoïdes ;

- coxa valga, grignotage de l'acetabulum.

\section{LES DIFFÉRENTES MPS}

Il y a un continuum entre les différentes formes cliniques.

\section{La MPS I}

- Déficit en alpha L iduronidase

- Fréquence $1 / 100000$ à $1 / 280000$ naissances

- 3 types classiques: qui sont un continuum : on distingue selon un ordre de sévérité décroissante[5]:

$\checkmark$ Hurler (retard mental)

$\checkmark$ Hurler-Scheie (intelligence normale)

$\checkmark$ Scheie (intelligence normale)

La maladie de Hurler se distingue par l'atteinte cérébrale avec notamment un retard mental : l'espérance de vie est inférieure à 10 ans (figure 2).

La maladie de Hurler Scheie est caractérisée par l'absence d'atteinte du SNC, une atteinte somatique plus tardive que dans la maladie de Hurler et une espérance de vie plus longue (figure 3).

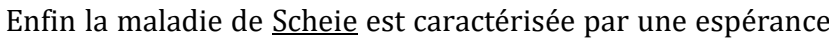
de vie beaucoup plus longue et une atteinte somatique très modérée.

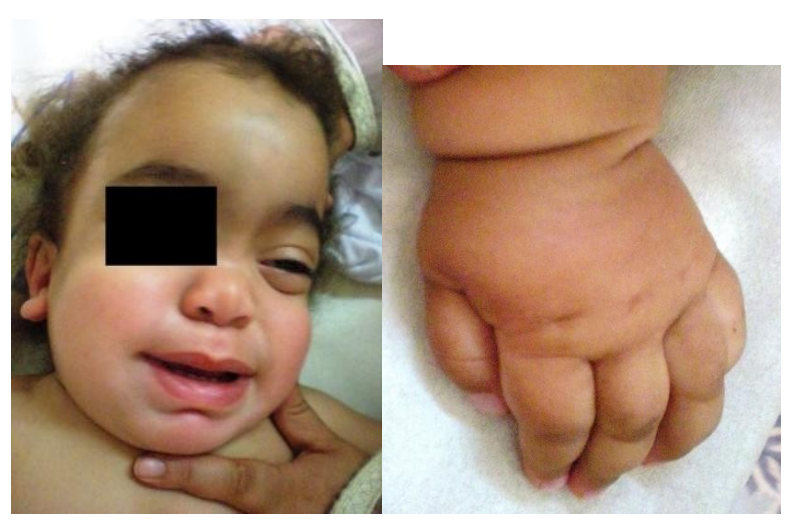

Figure 2 : Maladie de Hurler \{ l'âge de 3 ans, patiente décédée \{ l'âge de 6 ans par insuffisance cardiorespiratoire, noter le facies grossier et la main en griffe présents chez la plupart des patients atteints de MPS. 

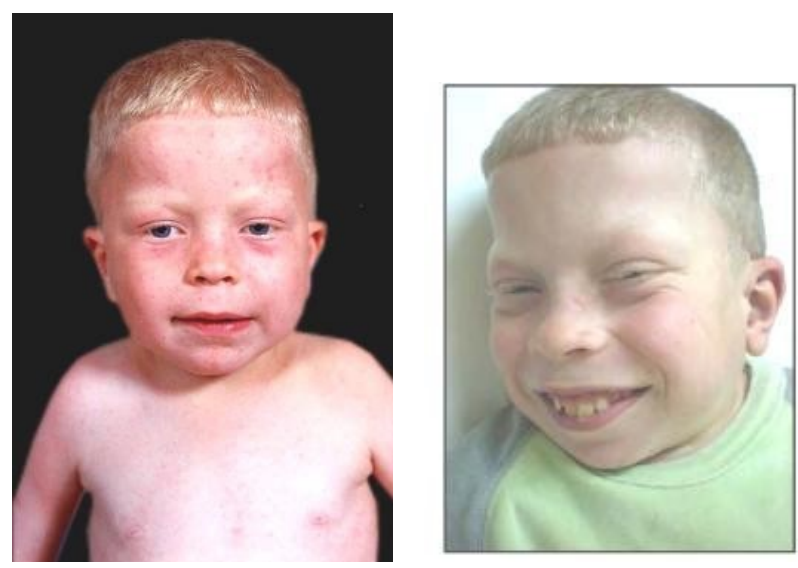

Figure 3 : Patient atteint de maladie de Hurler Scheie \{ l'âge de 6 ans et \{ l'âge de 11 ans. Noter l'épaississement progressif des traits après une période de 5 ans.

\section{La MPS II maladie de Hunter :}

Comme nous l'avons dit plus haut sa transmission se fait selon le mode lié \{ l'X.On retrouve \{ l'interrogatoire souvent des oncles maternels atteints.L'enzyme en cause est l'Iduronidase sulfatase nécessaire pour la dégradation de l'Héparane sulfate et le Dermatane sulfate. La fréquence est de 1/110 000 à $1 / 140000$ naissances [6]. Il existe 2 formes : une forme sévère avec retard mental et une forme atténuée avec une intelligence préservée.

$\checkmark$ La forme sévère a une espérance de vie limitée. Le décès survient généralement dans la 2èmedécennie alors que le patient est dans un état grabataire (figure 4).

$\checkmark$ La forme atténuée peut permettre une espérance de vie normale (Figure 4).

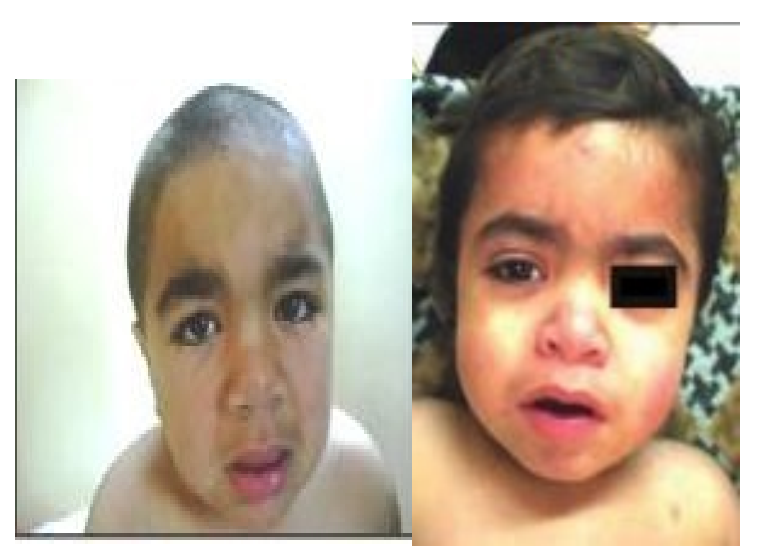

Figure 4 : Maladie de Hunter. à gauche forme atténuée ; à droiteforme sévère. Noter l'aspect grossier des traits chez les 2 patients, le traitement a été inefficace chez le $2^{\text {ème }}$ patient.

\section{La MPS III ou maladie de San Filippo :}

Il existe 4 différents types de la maladie selon le déficit enzymatique (figure 5) :

Type A : déficit en Héparane sulfatase ;

Type B : déficit en $\alpha$-N-acétyl glucosaminidase :

Type C : déficit en Acétyl-CoA- $\alpha$-glucosamine-transférase ;

Type D : déficit en $\mathrm{N}$-acétyl-glucosamine-6-sulfatase.

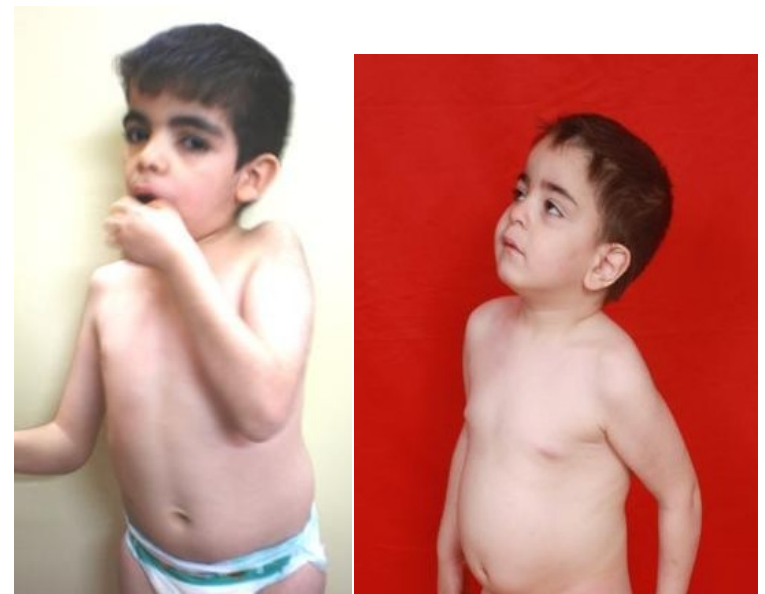

Figure 5 : à gauche MPS III type $\mathrm{A}$; à droite MPS III type B. Noter l'attitude inappropriée chez les 2 enfants, les sourcils épais chez le patient de gauche et l'absence de traits grossiers chez le patient de droite.

On ne peut pas différencier les 4 types sur des signes cliniques mais le type $\mathrm{A}$ semble le plus sévère. Le début survient entre 2 et 6 ans par des troubles du comportement et un retard d'acquisitions cognitives. Les manifestations respiratoires avec infections et troubles du sommeil attirent peu l'attention mais ils sont souvent associés \{ des troubles du comportement avec hyperactivité et tendance à l'automutilation. L'apparition de traits grossiers, si caractéristiques des MPS, sont très discrets au début ou même absents. On ne les met en évidence que tardivement [5].Par ailleurs, il n'y a pas de retard staturo-pondéral au cours des premières années, au contraire on retrouve fréquemment une avance staturale jusqu'\{ l'âge de 10 ans puis une décélération importante survient. La dysostose est discrète.

Le malade perd peu à peu toutes les acquisitions psychomotrices, le contrôle sphinctérien, et sa mobilité.La dégradation intellectuelle est inéluctable aboutissant au décès à la fin de la 2 èmedécennie ou au début de l'âge adulte, souvent lors d'une infection respiratoire.

\section{La MPS IV ou maladie de Morquio :}

Deux enzymes sont \{l'origine de la maladie : le IV $\mathbf{A}$ : dû à un déficit en $\mathrm{N}$-acétyl-galactosamine et le IV $\mathbf{B}$ \{un déficit en $\beta$ galactosidase.L'aspect clinique est le même pour les 2 types, il est caractéristique avec un nanisme avec raccourcissement du tronc, des opacités cornéennes minimes, une dysplasie osseuse différente des autres MPS et une préservation de l'intelligence (Figure 6) [7].

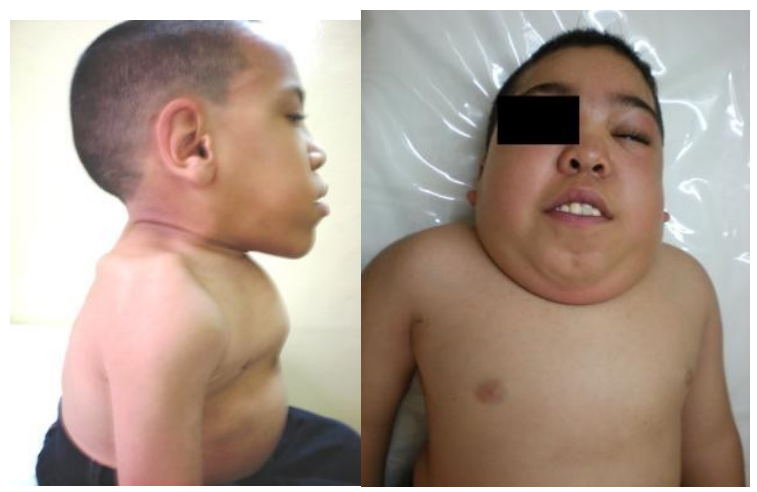

Figure 6 : MPS IV forme atténuée à gauche, forme grave à droite. Noter la protrusion sternale et l'hyperlaxité ligamentaire chez le patient de gauche, le patient de droite est décédé \{ l'âge de 14 ans des suites d'une insuffisance cardiorespiratoire. 
Les déformations osseuses sont différentes des autres MPS : genu valgum, hypoplasie de l'apophyse odontoïde \{l'origine de l'instabilité de la colonne et de blessures de la moelle épinière.La radiographie de la colonne lombaire montre des spicules $\{$ l'extrémité des corps vertébraux.

\section{La MPS VI ou maladie de Maroteaux Lamy}

C'est la moins fréquente des MPS, 1/340 000 naissances environ [8]. L'enzyme déficient est l'arylsulfatase.Il y a également 2 formes : une forme sévère avec atteinte somatique plus marquée et une forme atténuée, dans les 2 cas l'intelligence est préservée (figure 7) [5].

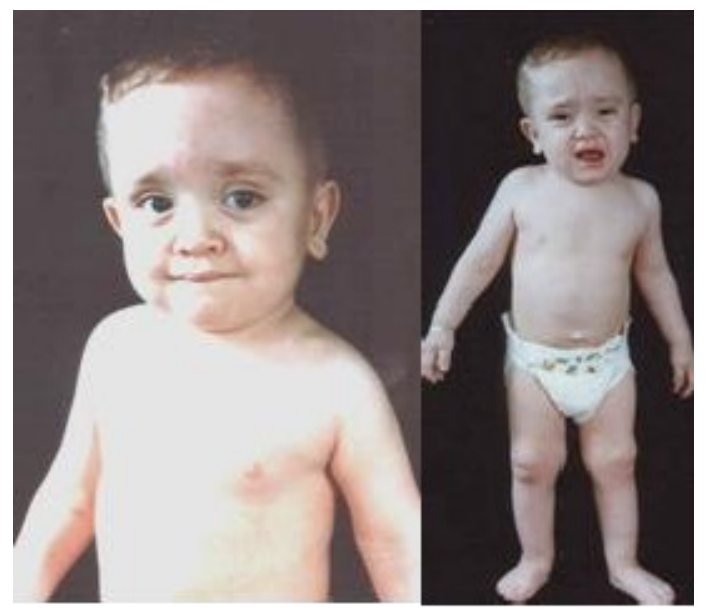

Figure 7 : MPS VI forme sévère : 2 frères atteints de la maladie actuellement grabataires en raison de coupures fréquentes du traitement

Les signes cliniques sont communs aux autres MPS avec une macrocrânie plus accentuée chez les patients atteints de MPS VI.

La MPS VII a les mêmes signes que la maladie de Hurler et ne peut être distinguée que par les études enzymatiques

La MPS IX est extrêmement rare et on n'a colligé que quelques patients dans le monde.

\section{PRISE EN CHARGE DES MPS}

L'Évaluation du degré de l'atteinte doit se faire à intervalles réguliers [9-11] :

1. Le syndrome d'obstruction des vois aériennes supérieures : épaississement de la langue et augmentation du volume des amygdales ;

2. Evaluation de la vision, de l'audition;

3. Recherche du syndrome du canal carpien (électromyogramme : les signes électriques précèdent les signes physiques) ;

4. Evaluation de la mobilité articulaire (goniomètre) des grosses articulations ;

5. Mesure du périmètre de marche durant 6 minutes $(6 \mathrm{mn}$ walk test);

6. Evaluation de l'atteinte cardiaque : échocardiographie, ECG : Il peut y avoir une atteinte des valvules mitrales et/ou aortiques, les artères coronaires sont le siège d'une obstruction parfois source d'ischémie. La myocardiopathieest la source principale de décès

7. L'atteinte de l'appareil respiratoire se manifeste par des déformations de la trachée \{l’origine de difficultés majeures lors de l'intubation pour anesthésie et d'autre part l'atteinte interstitielle est \{l'origine d'une hypo oxygénation.

8. La Recherche d'hydrocéphalie doit être systématique et peut nécessiter une dérivation ventriculo-péritonéale en cas d'hypertension intracrânienne. La compression spinale par épaississement de la dure-mère doit être surveillée par des examens successifs. Une laminectomie est indiquée si des signes cliniques apparaissent.

9. Nota Bene : L'anesthésie est un risque majeur pour les patients en raison de l'instabilité de l'atlas et de l'axis du fait de l'hypoplasie de l'apophyse odontoïde.

\section{Le diagnostic des MPS :}

Le diagnostic des MPS suspecté sur les signes cliniques, et est orienté par une analyse des GAG urinaires à la fois quantitative (augmentation et dans certaines formes très atténuées dans la plupart des cas mais pas toujours dans les MPS IV) et qualitative (mise en évidence de fraction anormales de dermatane sulfate, héparane sulfate et kératane sulfate permettant d'orienter le diagnostic (cf. tableau 2) $[12,13]$.

Tableau 2 : Synopsis du diagnostic pour les MPS

MPS I : Héparane Sulfate et Dermatane Sulfate (HS et DS)

MPS II: HS, DS

MPS III: HS

MPS IV: KS (Kératane sulfate)

MPS VI: DS

MPS VII : CS ou CS + DS + HS (CS= Chondroïtine sulfate) MPS IX : Acide Hyaluronique

Le diagnostic de certitude fait appel à des méthodes enzymatiques à la génétique $[14,15]$ :

- Recherche du déficit en cause sur des lymphocytes ou sur des fibroblastes.

- Actuellement le diagnostic enzymatique et éventuellement génétique peut se faire sur du papier buvard type "Guthrie » ou un papier filtre avec une bonne fiabilité [16].

- L'étude génétique peut mettre en évidence des génotypes susceptibles de traitement par greffe de moelle osseuse ou de par des cellules souches (comme dans la MPS I).

Le traitement symptomatique des MPS I-VII [17] fait appel à de nombreux spécialistes : Kinésithérapeute, cardiologue, et chirurgien cardiaque, ORL, Ophtalmologue, neurochirurgien, chirurgien, pneumologue.

Le traitement spécifique : Fait appel à 4 types de traitements :

La greffe de moelle osseuse, la greffe de cellules souches, l'enzyme recombinante et la thérapie génique.Un diagnostic précoce est impératif pour de meilleurs résultats car les séquelles sont généralement définitives.

L'enzyme de substitution est actuellement utilisée pour les MPS I, II, IV et VI. Elle est en cours d'évaluation pour les MPS III et VII.

MPS I : Aldurazyme (Laronidase) de Genzyme 100 $\mathrm{U} / \mathrm{Kg} /$ semaine ;

MPS II : Elaprase (Idursulfase) de Shire $0,5 \mathrm{mg} / \mathrm{kg}$, et Hunterase (Idursulfase béta) de Green Cross à la même dose approuvée par la FDA et actuellement utilisée en Algérie et 
dans certains autres pays ;

MPS IV : Vimizim (Elosulfase alpha) utilisée aux USA et actuellement en Europe ;

MPS VI : Naglazyme (Galsulfase) par Biomarin à la dose de 1 $\mathrm{mg} / \mathrm{kg}$.

Greffe de moelle ou de cellules souches: Avantages : elle prévient l'atteinte cérébrale si utilisée tôt dans la MPS I avec génotypes : W402X et Q70X. Ces génotypes sont associés à une atteinte cérébrale si le traitement par la greffe ne se fait pas suffisamment tôt (avant 2 ans). Amélioration de la fonction cardiaque, respiratoire, articulaire, de l'audition, de la vision, diminution de l'hépatosplénomégalie.Inconvénients : risque de décès augmentés, risque de rejet.

Les enzymes recombinantes ou de substitution: avantages : amélioration de la mobilité articulaire, du périmètre de marche, de la fonction respiratoire, réduction de l'hépatosplénomégalie.Effets secondaires bénins : céphalées, fièvre, urticaire, effets secondaires graves : risque de réaction anaphylactique nécessitant un traitement d'urgence, troubles respiratoires.

La thérapie génique est actuellement utilisée dans certains centres (Bicêtre : Professeur Marc Tardieu : maladie de San Filippo B) : il s'agit de l'utilisation d'un virus génétiquement modifié et introduit dans le cerveau du patient. Le traitement n'est utilisé que chez des patients diagnostiqués par dépistage de la maladie dans la famille d'un patient atteint. Ce traitement doit être fait avant l'apparition des signes. Ce traitement est en cours d'essai clinique.

\section{CONCLUSION}

Les mucopolysaccharidoses sont des maladies rares mais compte tenu du contexte de consanguinité dans lequel nous vivons en Algérie, la fréquence est sûrement sous-estimée par manque d'information auprès des médecins généralistes. Un effort doit être fait à ce niveau pour détecter cette pathologie plus précocement et permettre un traitement rapide. Il est indispensable que les autorités fassent plus de facilités pour l'obtention des médicaments lorsque le diagnostic est établi et surtout qu'il n'y ait plus de rupture de stock comme c'est souvent le cas actuellement.

Par ailleurs, le traitement doit se faire au début par des équipes habituées à ce type de pathologie. Les malades doivent ensuite être transférés dans des hôpitaux au plus près de leur zone d'habitation pour éviter les déplacements fatigants pour les parents et les patients. Un consensus à l'échelle nationale est plus que souhaitable pour choisir la meilleure stratégie à prendre et également pour que les ressources financières soient utilisées le mieux possible, car le traitement de ces pathologies est extrêmement coûteux.
Déclaration d'intérêts : l'auteur ne déclare aucun conflit d'intérêt en rapport avec cet article.

\section{Messages clés}

maladies lysosomales faciles $\{$ détecter pour la plupart d'entre elles par des signes communs : faciès grossier, cou court, gibbus, hépatosplénomégalie, retard staturopondéral, atteinte cérébrale avec régression psychomotrice chez certaines MPS.

$\checkmark$ Possibilité de traitement chez un grand nombre d'entre elles (greffe de moelle, enzymes de substitution, thérapie génique, et traitement des atteintes viscérales exemple adénoïdectomie, chirurgie cardiaque etc.)

\section{RÉFÉRENCES}

1. Baloghova J ; Chief Editor: Elston DM. Mucopolysaccharidoses Type I-VII : emedicine.medscape.com/article/1115193-overview

2. Mc Kusick VA. The Genetic mucopolysaccharidoses. Medicine 44:445-470, 1965

3. A Le Treut. Les glucides. Faculté de Rennes 1 Cours de Biochimie pour CPEM.

4. Andrieu N, Codogno P, Levade T. Les Lysosomes: Atlas des maladies lysosomales Vaincre les maladies lysosomales Généralités.

5. Neufeld ER, Muenzer J. The Mucopolysaccharidosis : in Scriver CR, Beaudet AL, Sly WS, The metabolic and Molecular Bases of Inherited Diseases 7 th ed. New York, Mc Graw-Hill, 1995, 3421-3451

6. Lowry RB, Applegarth DA, Toone JR, et al. Update on frequency of Mucopolysaccharide syndrome In British Columbia. Human genetic : 85:389, 1990

7. Montano AM, Tomatsu S, Gottesman GS, et al. International Morquio Registry: clinical manifestation and natural course of Morquio A disease. inherited Metab Dis. Apr 2007;30(2):165-14

8. Ben Turkia H, Tebib N, Azzouz H, et al. Incidence des mucopolysaccharidoses en Tunisie. La Tunisie Médicale 2009; Vol 87( $\left.n^{\circ} 11\right)$ : 782-785

9. Muenzer J, Beck M, Eng CM, et al.Multidisciplary management of Hunter Disease. Pediatrics 2009; 124: e1228-39

10. Muenzer J, Wraith JE, Clarke LA. Mucolysaccharidosis: management and treatment guidelines. Pediatrics 2009; 123: 19-29

11. Valayanopoulos V, Nicely $\mathrm{H}$, Harmatz $\mathrm{P}$, et Al. Mucopolysaccharidosis VI. Orphanet Journal of Rare Diseases $2010 ; 5: 5$

12. Pennock CA. A review and selection of simple laboratory methods used for the study of glycosaminoglycan excretion and the diagnosis of the mucopolysaccharidoses. J Clin Pathol 29:111, 1976

13. Piraud M, Boyer S, Mathieu J, et al.Diagnosis of Mucopolysaccharidoses in a clinically selected population by urinary GAG analysis : a study of 2000 urine samples. Clinica Chimica Acta 1993, vol. 221(1)-171-81

14. Hall CW, Liebaers I, Di Natale $P$, et al. Enzymic diagnosis of the genetic mucopolysaccharide storage disorders. Methods Enzymol $50: 439,1978$

15. Kresse $\mathrm{H}$, Von Figura $\mathrm{K}$, Klein $\mathrm{U}$, et al. Enzymic diagnosis of the genetic mucopolysaccharide storage disorders. Methods Enzymol 83: 559, 1982

16. Gelb MH, Turecek F, Scott $\mathrm{CR}$, et al. Direct multiplex assay of enzymes in dried blood spots by tandem spectrometry for the new born sreening of lysosomale storage disorders. J Inherit Metab Dis 2006; 29 : 397-404

17. Baloghova J, Schwartz RA, Zuzana Baranova, et al. Mucoplolysaccharidoses Types I-VII: treatment \& management. emedicine. Medscape.com/article/ 1115193-treatment

Cet article a été publié dans le « Batna Journal of Medical Sciences » BJMS, I'organe officiel de "L'association de la Recherche Pharmaceutique - Batna »

Le contenu de la Revue est ouvert « Open Access » et permet au lecteur de télécharger, d'utiliser le contenu dans un but personnel ou d'enseignement, sans demander l'autorisation de l'éditeur/auteur. 